13

\title{
Вероятность ионизации атомов, распыленных при бомбардировке поверхности металлов одно- и многозарядными ионами
}

\author{
(C) С.Ф. Белых, ${ }^{1}$ А.Д. Беккерман ${ }^{2}$ \\ ${ }^{1}$ Московский авиационный институт (национальный исследовательский университет), \\ 125993 Москва, Россия \\ ${ }^{2}$ Schulich Faculty of Chemistry, Technion - Israel Institute of Technology, \\ 32000 Haifa, Israel \\ e-mail: serolg@rambler.ru
}

Поступило в Редакцию 17 июля 2021 г.

В окончательной редакции 27 сентября 2021 г.

Принято к публикации 12 октября 2021 г.

Исследованы процессы ионизации атомов, распыленных при бомбардировке чистой поверхности металлов одно- и многозарядными ионами с одинаковой кинетической энергией порядка нескольких $\mathrm{keV}$. В рамках феноменологической модели образования атомных ионов, учитывающей релаксацию локального возбуждения электронов в металле, получены аналитические формулы для расчета вероятности ионизации распыленных атомов. Показано, что по сравнению с однозарядными ионами бомбардировка металла многозарядными ионами приводит к существенному росту вероятности ионизации распыленных атомов за счет более эффективного возбуждения электронов и увеличения времени релаксации этого возбуждения.

Ключевые слова: ионное распыление, вероятность ионизации, многозарядные ионы, распыленные атомы, оже-электроны, каскады столкновений, масс-спектрометрия вторичных ионов.

DOI: 10.21883/JTF.2022.02.52023.218-21

\section{Введение}

Бомбардировка чистой поверхности металлов (под чистой понимается поверхность металла, свободная от адсорбированных атомов и молекул) однозарядными атомными и кластерными ионами инициирует эмиссию атомов и кластеров в различных зарядовых состояниях [1-4]. Использование эмиссии атомных ионов лежит в основе различных модификаций метода масс-спектрометрии вторичных ионов (МСВИ) [5,6]. Однако эффективность генерации атомных ионов относительно мала (в среднем из десяти тысяч распыленных атомов образуется всего лишь несколько ионов), что стимулирует поиск путей ее усиления. Такая возможность возникает при бомбардировке металлов многозарядными ионами (МЗИ). Недавно было установлено, что по сравнению с однозарядными ионами взаимодействие МЗИ с поверхностью металлов приводит к существенному увеличению интенсивности выхода распыленных атомных ионов [7,8] и возбужденных атомов [9]. Эти результаты свидетельствуют о возможности повышения чувствительности, а следовательно, и информативности методов диагностики поверхности при использовании МЗИ в качестве зондирующих пучков. Для практической реализации такого подхода в методе МСВИ необходимо знание физических процессов, происходящих при взаимодействии одно- и многозарядных ионов с поверхностью металлов, которое позволит ответить на вопрос - почему интенсивность выхода атомных ионов относительно мала при использовании однозарядных атомных и кластерных ионов и существенно велика при использовании МЗИ.

В настоящей работе приведен анализ причин, ограничивающих генерацию атомных ионов при бомбардировке чистой поверхности металлов однозарядными атомными и кластерными ионами. Предложена простая феноменологическая модель образования атомных ионов, учитывающая процесс релаксации локального электронного возбуждения в металле. В рамках такой модели проведено сравнительное исследование вероятности ионизации атомов, распыленных с чистой поверхности металла одно- и многозарядными ионами с одинаковой кинетической энергией порядка нескольких $\mathrm{keV}$.

\section{1. Анализ причин, ограничивающих генерацию атомных ионов при бомбардировке чистой поверхности металлов однозарядными атомными и кластерными ионами}

Выделим причины, ограничивающие генерацию атомных ионов, анализируя результаты исследования эмиссии атомных и кластерных ионов при бомбардировке Та и $\mathrm{Nb}$ однозарядными атомными $\mathrm{Au}^{-}$и кластерными $\mathrm{Au}_{3}^{-}$ ионами с энергией $6 \mathrm{keV} /$ atom [3].

Атомные ионы $6 \mathrm{keV} \mathrm{Au}^{-}$распыляют металлы в режиме линейных каскадов столкновений, характеризующихся малой плотностью движущихся атомов в 
объеме каскада [2]. Распыление реализуется, когда движущийся атом передает неподвижному атому поверхности импульс, достаточный для отрыва от металла. В этих условиях время эволюции каскада $t_{0} \sim 10^{-12} \mathrm{~s}$, коэффициент распыления $S \sim 4$ atom/ion [10], а состав распыленных частиц представлен в основном атомами. Зарядовое состояние атомов формируется на отлете от металла (на расстоянии $\sim 3-5 \AA$ ) путем электронного обмена с поверхностью (см., например, [11] и ссылки в этой работе). Вблизи металла валентный уровень $\varepsilon_{a}$ атома расположен напротив заполненной части зоны проводимости и электронный обмен возможен только при возбуждении электронов (образовании вакансий на уровнях энергии $\varepsilon$, лежащих ниже уровня Ферми $\varepsilon_{F}$ ). Возбуждение в объеме каскада возникает при передаче электронам части кинетической энергии от бомбардирующего иона и быстрых атомов отдачи [12-14]. В металлах за время $\tau \sim 10^{-14}$ s происходит релаксация возбуждения за счет процессов электропроводности и рекомбинации электронов с вакансиями [15-17]. Поэтому вероятность ионизации атомов $P^{+}$мала $\left(\sim 10^{-4}\right)$ : ионизируются только атомы, покидающие поверхность с большой скоростью на ранних стадиях эволюции каскада столкновений. Таким образом, в режиме линейных каскадов быстрая релаксация электронного возбуждения ограничивает генерацию атомных ионов.

Кластерные ионы $18 \mathrm{keV} \mathrm{Au} \mathrm{Au}_{3}^{-}$распыляют металлы в режиме нелинейных каскадов, имеющих высокую плотность движущихся атомов $[18,19]$. Нелинейный каскад формируется ко времени $t_{0} \sim 10^{-11} \mathrm{~s}$ после затухания линейных каскадов, когда энергия движущихся атомов уже недостаточна для отрыва неподвижных атомов от металла. В последующих столкновениях движущиеся атомы перераспределяют свою энергию среди большой совокупности атомов в объеме каскада. В этих условиях реализуются иные механизмы распыления и ионизации атомов. Если „температура $T_{\text {eff “ }}\left(T_{\text {eff }}\right.$ характеризует среднюю энергию, приходящуюся на один движущийся атом в объеме каскада) меньше критической температуры $T_{\text {cr }}$ металла, то происходит испарение атомов из локального участка поверхности [20]. При $T_{\text {eff }}>T_{\text {cr }}$ металл в объеме каскада претерпевает фазовый переход и превращается в пар. Оценка показывает, что в рассматриваемом случае распыления Та пар имеет начальную температуру $T_{\text {eff }} \sim 37000 \mathrm{~K}$, концентрацию атомов $n \sim 5 \cdot 10^{22} \mathrm{~cm}^{-3}$ и давление $P \sim 28 \mathrm{GPa}$ [19]. За счет перепада давлений пар вырывается в вакуум, формируя поток частиц. В этом случае коэффициент распыления $S\left(S>10^{3}\right)$ грубо оценивается из числа атомов $(\sim 2000)$ в объеме каскада [19]. При фазовом переходе исчезает поверхность (граница раздела металл-вакуум), и в потоке пара ионы образуются за счет механизма ударной ионизации [21]. В этом случае вероятности ионизации $P^{+}$оцениваются по формуле Саха [22] и на начальном этапе эволюции потока пара величина $P^{+} \sim 0.1[21]$.
Сравнение значений $S$ и $P^{+}$для разных режимов показывает, что в режиме нелинейных каскадов процессы распыления и ионизации атомов гораздо более эффективны, что должно приводить к интенсивной эмиссии атомных ионов. Однако результаты [3] свидетельствуют о том, что при переходе от линейного к нелинейному режиму распыления интенсивность выхода атомных ионов увеличивается всего лишь в несколько раз, тогда как сумма интенсивностей выхода кластерных ионов возрастает более чем в 100 раз. Это указывает на то, что образующиеся в потоке пара атомные ионы играют роль центров конденсации атомов в кластеры. Конденсация происходит вплоть до расстояния $\sim 5000 \AA$ от поверхности (до тех пор, пока не прекратятся столкновения частиц в потоке) и приводит к перераспределению частиц в пользу кластерных ионов [19]. Таким образом, в режиме нелинейных каскадов генерация атомных ионов ограничена процессом конденсации. Частичное либо полное снятие указанных ограничений должно приводить к усилению генерации атомных ионов. Такая возможность возникает при распылении металлов многозарядными ионами, что впервые было продемонстрировано в работе [7], где было найдено, что по сравнению с ионами

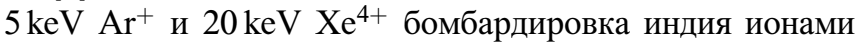
$20 \mathrm{keV} \mathrm{Xe}{ }^{40+}$ приводит к существенному росту вероятности ионизации распыленных атомов и увеличению вклада медленных атомов в процесс ионизации. В связи с этим представляет интерес сравнительное исследование процессов ионизации атомов, распыленных с чистой поверхности металла одно- и многозарядными ионами данного химического элемента с одинаковой кинетической энергией $\sim$ нескольких $\mathrm{keV}$. Такое исследование проведено в рамках простой феноменологической модели образования ионов, учитывающей релаксацию электронного возбуждения в металле.

\section{2. Модель образования атомных ионов}

Предлагаемый подход является развитием модели образования ионов $[23,24]$, основанной на следующих предположениях:

(a) бомбардировка металлов однозарядными атомными ионами вызывает, помимо распыления атомов в режиме линейных каскадов столкновений, локальное возбуждение электронов (см. [12-14] и ссылки в этих работах) с постоянной „электронной температурой $T_{e}$ “, причем $T_{e}$ не является температурой в термодинамическом смысле этого слова, а используется как параметр, характеризующий неравновесное распределение электронов по энергии в объеме каскада столкновений;

(b) зарядовое состояние атома формируется на отлете от металла за счет туннельного перехода электрона с валентного уровня $\varepsilon_{a}$ атома на незаполненный уровень $\varepsilon$ зоны проводимости. 
В [23,24] вероятность ионизации $P^{+}$распыленного атома дается выражением:

$$
P^{+}=\exp \left[\frac{\varepsilon_{a}\left(z_{0}\right)-\varepsilon_{F}}{k_{B} T_{e}}\right],
$$

где $z_{0}=(1 / \gamma) \ln \left(2 \Delta_{0} \hbar \gamma V_{z}\right)$ - расстояние до поверхности („freezing“ distance [25]) - при $z<z_{0}$ зарядовое состояние атома близко к равновесному, а за пределами этой области электронный обмен прекращается; $2 \Delta_{0}-$ ширина валентного уровня $\varepsilon_{a}$ при $z=0$ : $\varepsilon_{F}-$ уровень Ферми; $1 / \gamma$ - расстояние, на котором величина $2 \Delta_{0}$ убывает в $e$ раз; $V_{z}$ - нормальная составляющая скорости атома; $\hbar$ и $k_{B}$ - постоянные Планка и Больцмана.

В $[23,24]$ постулат о постоянстве значения $T_{e}$ противоречит реальности, поскольку в процессе релаксации электронного возбуждения текущая температура $T_{t}$ убывает от $T_{e}$ до $T \approx 0$. Поэтому в дальнейшем будет использована формула (1), модифицированная путем замены постоянного значения $T_{e}$ на текущую температуру $T_{t}$. Введем следующие дополнительные предположения:

(i) вакансии, генерируемые на начальном этапе эволюции каскада в 2-3 приповерхностных слоях металла, играют определяющую роль в электронном обмене атомов с поверхностью;

(ii) при $t=0$ (момент прекращения генерации вакансий в приповерхностных слоях) возбуждение соответствует неравновесному распределению электронов по энергии $f_{e}$ при температуре $T_{e}$. В процессе релаксации возбуждения $f_{e}$ стремится к равновесному распределению $f_{F}\left(f_{F}-\right.$ функция Ферми при $\left.T \cong 0\right)$.

Для нахождения зависимости $T_{t}$ от времени $t$ воспользуемся простейшей формой кинетического уравнения Больцмана:

$$
\frac{\partial f_{t}}{\partial t}=-\left(f_{t}-f_{F}\right) \frac{1}{\tau}
$$

где $f_{t}$ - неравновесное распределение электронов по энергии при температуре $T_{t}$ к моменту времени $t, \tau-$ время релаксации.

При $f_{F}=1(T \cong 0)$ и $\left(\varepsilon-\varepsilon_{F}\right)<0$ решение уравнения (2) имеет вид

$$
1-f_{t}=\left(1-f_{e}\right) \exp \left(-\frac{t}{\tau}\right)
$$

Насколько нам известно, в литературе отсутствуют какие-либо сведения о виде функций $f_{e}$ и $f_{t}$, поэтому в качестве грубого приближения в формуле (3) заменим эти функции функциями Ферми при температурах $T_{e}$ и $T_{t}$ соответственно. Тогда при $\varepsilon<\varepsilon_{F}$ и $\left(\varepsilon_{F}-\varepsilon\right)>k_{B} T$ из формулы (3) можно получить зависимость температуры $T_{t}$ от времени $t$

$$
\frac{1}{T_{t}}=\frac{1}{T_{e}}+\frac{t k_{B}}{\tau\left(\varepsilon_{F}-\varepsilon\right)} .
$$

Согласно формуле (4), вероятность ионизации $\mathrm{P}^{+}$ зависит от $t=t^{*}+t_{s}$, где $t^{*}=z_{0} / V_{z}-$ время движения атома от поверхности $(z=0)$ до $z=z_{0}$ и $t_{s}\left(0<t_{s}<t_{0}\right)$ - эмиссионное время (момент отрыва атома от поверхности). Поток распыленных атомов характеризуется скоростным $F\left(V_{z}\right)$ и частотным $G\left(t_{s}\right)$ распределениями. В работе [26] показано, что для системы $5 \mathrm{keV} \mathrm{Ar}{ }^{+} / \mathrm{Cu}$ частотное распределение $G\left(t_{s}\right)$ хорошо аппроксимируется функцией $G\left(t_{s}\right)=\bar{t}_{s}^{-2} \exp \left(-t_{s} / \bar{t}_{s}\right) t_{s}$, где $\bar{t}_{s} \approx 2.5 \cdot 10^{-13} \mathrm{~s}-$ наиболее вероятное время эмиссии. В зависимости от $t^{*}$ и $t_{s}$ распыленные атомы, достигая расстояния $z_{0}$, взаимодействуют с локальным участком поверхности, имеющим различные значения текущей температуры $T_{t}$. При данном значении $t_{s}$ быстрые и медленные атомы „наблюдают“ большую и меньшую температуру $T_{t}$ соответственно. Энергию валентного уровня $\varepsilon_{a}(z)$ представим в виде $\varepsilon_{a}(z)=\varepsilon_{a}(\infty)+e^{2} / 4 z$ (e - заряд электрона), что справедливо при $z>a$, где $a-$ постоянная решетки [23]. Подставляя выражение (4) и приближенное значение $\varepsilon_{a}(z)$ в модифицированную формулу (1), получим выражение для расчета вероятность ионизации $P^{+}\left(V_{z}, t_{s}\right)$ атомов, распыленных из металла однозарядными ионами

$P^{+}\left(V_{z}, t_{s}\right) \cong \exp \left\{-\left[\frac{\left(I-\varphi-e^{2} / 4 z_{0}\right)}{k_{B} T_{e}}+\left(t_{s}+\frac{z_{0}}{V_{z}}\right) \frac{1}{\tau}\right]\right\}$.

Здесь $\varepsilon_{a}(\infty)$ и $\varepsilon_{F}$ принимаются равными соответственно энергии ионизации атома $I$ и работе выхода чистой поверхности металла $\varphi$. Обе энергии отсчитываются от уровня вакуума.

Рассмотрим особенности взаимодействия многозарядных ионов с металлом, полагая при этом, что одно- и многозарядные ионы принадлежат одному химическому элементу и имеют одинаковую кинетическую энергию $E_{0}$ (при задании $E_{0}$ необходимо внести поправку на ускорение МЗИ в поле сил зеркального изображения $[27,28])$.

МЗИ обладают запасом потенциальной энергии, величина которой зависит от кратности заряда иона $q$ и равна сумме энергий удаления $q$ электронов (например, потенциальные энергии ионов $\mathrm{Dy}^{8+}, \mathrm{Dy}^{10+}$ и $\mathrm{Dy}^{12+}$ равны 453, 764 и $1159 \mathrm{eV}$ соответственно [29]). Вблизи металла (при $z \sim 10 \AA$ ) происходит нейтрализация МЗИ за счет резонансных переходов электронов металла на возбужденные уровни иона с образованием высоковозбужденного атома („hollow atom“ $[27,28]$ ). Последующий распад возбужденного состояния атома путем каскада спонтанных оже-переходов инициирует изотропное испускание быстрых оже- электронов. Примерно половина оже-электронов проникает в металл и в процессе электрон-электронных столкновений передает электронам металла энергию, приблизительно равную половине потенциальной энергии МЗИ, вызывая в локальном элементе объема металла возбуждение, характеризуемое температурой $T_{e}^{*}$. Помимо возбуждения, характеризуемого температурой $T_{e}^{*}$, взаимодействие МЗИ с металлом приводит, как и в случае однозарядных ионов, к распылению атомов в режиме линейных каскадов столкновений 
и возбуждению электронов, соответствующее температуре $T_{e}$. Возбуждение реализуется более эффективно при электрон-электронных, чем при атом-электронных столкновениях, и потому разумно полагать, что $T_{e}^{*}>T_{e}$ при $q \gg 1$. Оценка показала, что указанные электронные возбуждения синхронизированы во времени и локализованы в пределах участка поверхности, размеры которого не превышают поперечное сечение каскада столкновений. Это дает основание полагать, что многозарядные ионы формируют начальное (при $t=0$ ) возбуждение, соответствующее температуре $T_{e}^{*}+T_{e}$.

При приложении к поверхности металла положительного потенциала $U_{0}$ (такой подход используется в масс-спектрометрии вторичных ионов при регистрации положительных ионов) оже-электроны, движущиеся от поверхности, под действием тормозящего электрического поля возвращаются в металл и, взаимодействуя с электронами металла, формируют последовательность запаздывающих импульсов возбуждения. Время запаздывания таких импульсов определяется величиной $U_{0}$. На формирование запаздывающих импульсов возбуждения тратится энергия, приблизительно равная половине потенциальной энергии МЗИ. Наложение последовательности запаздывающих импульсов возбуждения на начальное (при $t=0)$ возбуждение приводит к более медленному распаду электронного возбуждения, что эквивалентно увеличению времени релаксации $\tau^{*}\left(\tau^{*}>\tau\right)$.

Учитывая вышесказанное, выражение для расчета вероятности ионизации $P^{q+}\left(V_{z}, t_{s}\right)$ атомов, распыленных при бомбардировке металлов многозарядными ионами, представим в виде

$$
\begin{aligned}
P^{q+}\left(V_{z}, t_{s}\right) \cong & \exp \left\{-\left[\frac{\left(I-\varphi-e^{2} / 4 z_{0}\right)}{k_{B}\left(T_{e}+T_{e}^{*}\right)}\right.\right. \\
& \left.\left.+\left(t_{s}+\frac{z_{0}}{V_{z}}\right) \frac{1}{\left(\tau+\tau^{*}\right)}\right]\right\} .
\end{aligned}
$$

\section{3. Обсуждение полученных результатов}

Из сравнения формул (5) и (6) при данных значениях $t_{s}$ и $V_{z}$ следует, что бомбардировка металла многозарядными ионами приводит к существенному увеличению вероятности ионизации распыленных атомов $P^{q+}\left(V_{z}, t_{s}\right)>P^{+}\left(V_{z}, t_{s}\right)$.

На рис. 1 приведены рассчитанные по формулам (5) и (6) зависимости вероятностей ионизации $P^{+}\left(V_{z}, t_{s}\right)$ и $P^{q+}\left(V_{z}, t_{s}\right)$ от скорости распыленных атомов тантала при трех значениях температуры $T_{e}^{*}(0,1000$ и $3000 \mathrm{~K})$ и фиксированном значении $t_{s}=5 \cdot 10^{-14} \mathrm{~s}$. Потенциал поверхности металла на рис. 1,a принимался равным $U_{0}=0$, а на рис. $1, b-U_{0}=1 \mathrm{kV}$. В расчетах использованы следующие значения параметров: $\gamma=1.25 \cdot 10^{8} \mathrm{~cm}^{-1}[14], 2 \Delta_{0}=5 \mathrm{eV}[14], I=7.5 \mathrm{eV}$, $\varphi=4.2 \mathrm{eV}, \quad \tau=5 \cdot 10^{-14} \mathrm{~s} \quad[16,17], \quad \tau^{*}=1 \cdot 10^{-14} \mathrm{~s}$ и
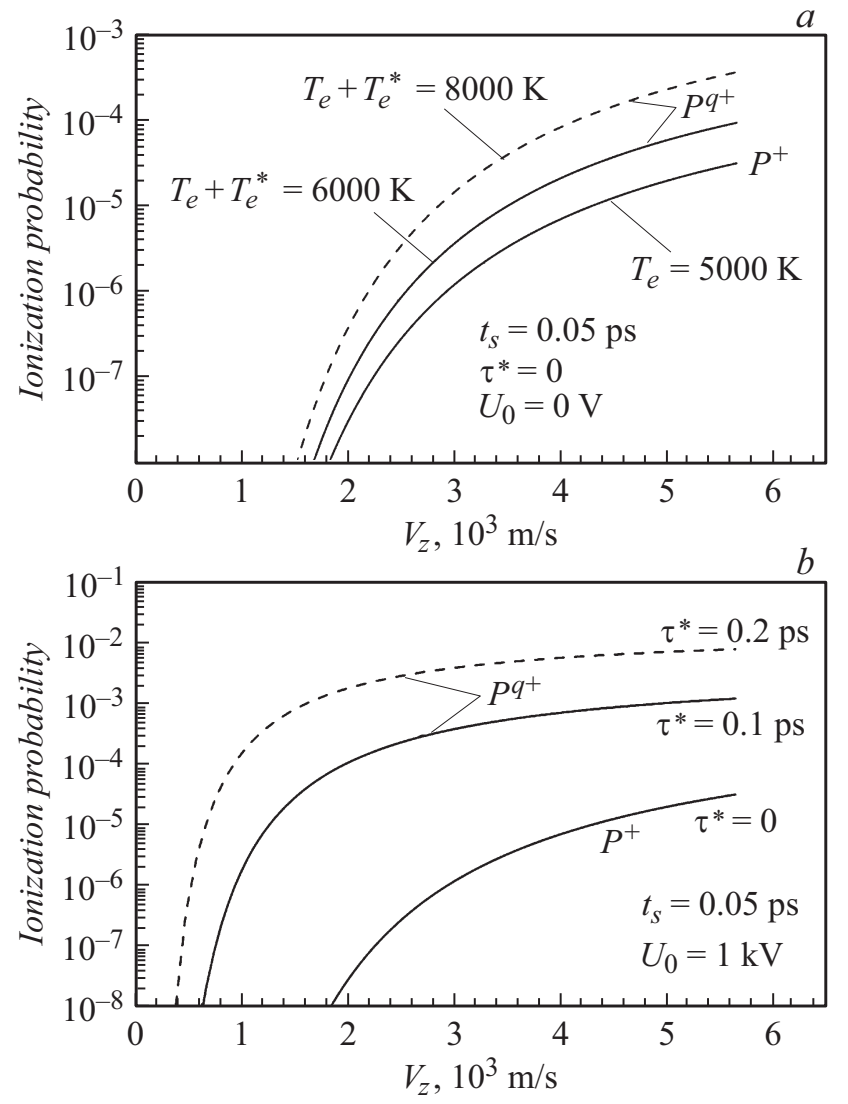

Рис. 1. $a-$ зависимости вероятностей ионизации $P^{+}\left(V_{z}, t_{s}\right)$ и $P^{q+}\left(V_{z}, t_{s}\right)$ от скорости $V_{z}$ атомов тантала, распыленных однои многозарядными ионами при трех значениях температуры $T_{e}^{*}-0,1000$ и $3000 \mathrm{~K}$ и фиксированных значениях параметров $T_{e}=5000 \mathrm{~K}, \tau^{*}=0, \tau=5 \cdot 10^{-14} \mathrm{~s} \mathrm{и} t_{s}=5 \cdot 10^{-14} \mathrm{~s}$. Потенциал распыляемой поверхности тантала $U_{0}=0 ; b-$ зависимости вероятностей ионизации $P^{+}\left(V_{z}, t_{s}\right)$ и $P^{q+}\left(V_{z}, t_{s}\right)$ от скорости атомов тантала для потенциала $U_{0}=1 \mathrm{kV}$ (режим генерации запаздывающих импульсов возбуждения электронной подсистемы металла при $\tau^{*}=10^{-13}$ и $2 \cdot 10^{-13} \mathrm{~s}$ ).

$2 \cdot 10^{-13} \mathrm{~s}, T_{e}=5000 \mathrm{~K}$. Из рис. $1, a$ следует, что с ростом температуры $T_{e}^{*}\left(T_{e}^{*}\right.$ зависит от потенциальной энергии МЗИ, т. е. от кратности заряда $q$ ) происходит увеличение вероятности ионизации $P^{q+}\left(V_{z}, t_{s}\right)$ распыленных атомов в диапазоне значений скорости $V_{z}(0.5-6) \cdot 10^{3} \mathrm{~m} / \mathrm{s}$. При этом возрастание величины $P^{q+}\left(V_{z}, t_{s}\right)$ происходит за счет увеличения вклада в процесс ионизации как быстрых, так и медленных распыленных атомов. Гораздо более сильный эффект увеличения вероятности ионизации $P^{q+}\left(V_{z}, t_{s}\right)$ наблюдается при приложении к поверхности металла положительного потенциала $U_{0}=1 \mathrm{kV}$ (рис. $1, b)$. В этом случае за счет увеличения времени релаксации электронного возбуждения $\tau^{*}\left(\tau^{*}>\tau\right)$ происходит существенный рост вклада медленных атомов в процесс ионизации.

На рис. 2 приведены зависимости вероятностей ионизации $P^{+}\left(V_{z}, t_{s}\right)$ и $P^{q+}\left(V_{z}, t_{s}\right)$ от скорости $V_{z}$ распыленных атомов тантала, рассчитанные при различных 


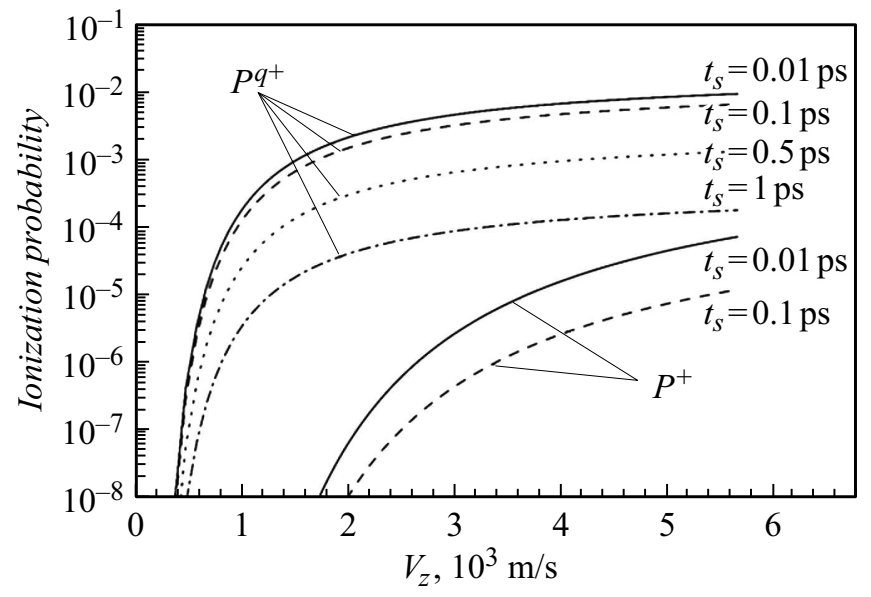

Рис. 2. Зависимости вероятностей ионизации $P^{+}\left(V_{z}, t_{s}\right)$ и $P^{q+}\left(V_{z}, t_{s}\right)$ от скорости атомов тантала при температурах $T_{e}^{*}=0 \mathrm{~K}$ и $3000 \mathrm{~K}$ и времени $t_{s}=10^{-14}, 10^{-13}, 5 \cdot 10^{-13}$ и $10^{-12} \mathrm{~s}$. Использованы фиксированные значения параметров $T_{e}=5000 \mathrm{~K}, \tau=5 \cdot 10^{-14} \mathrm{~s}$ и $\tau^{*}=0$ и $2 \cdot 10^{-13} \mathrm{~s}$ при потенциале распыляемой поверхности тантала $U_{0}=1 \mathrm{kV}$.

значениях времени отрыва атома от поверхности металла $t_{s}-1 \cdot 10^{-14}, 1 \cdot 10^{-13}, 5 \cdot 10^{-13}$ и $1 \cdot 10^{-12} \mathrm{~s}$. Как следует из рис. 2, при бомбардировке металла многозарядными ионами помимо быстрых атомов эффективно ионизуются и медленные атомы, распыленные на поздних стадиях эволюции линейного каскада столкновений и соответственно локального электронного возбуждения. При бомбардировке металлов однозарядными ионами эффективность ионизации мала - ионизуются в основном только быстрые атомы, распыленные на ранних стадиях эволюции каскада столкновений. Отметим, что в предлагаемой модели в качестве грубого приближения была проведена замена неизвестных неравновесных функций распределения электронов по энергии в локальной области возбуждения функциями Ферми. В пользу такого подхода говорит то, что модель не только качественно описывает (в согласии с результатами [7]) эффект увеличения вероятности ионизации атомов, распыленных при бомбардировке металла многозарядными ионами, но и поясняет особенности процесса ионизации быстрых и медленных атомов на ранних и поздних стадиях эволюции линейного каскада столкновений и электронного возбуждения (рис. 1 и 2). Следует указать, что предлагаемая модель пригодна также для предсказания и описания особенностей малоизученных либо совсем не изученных процессов эмиссии возбужденных атомов и отрицательных ионов при бомбардировке металлов многозарядными ионами.

\section{Заключение}

В работе проведен анализ физических процессов, приводящих к эмиссии атомных ионов при бомбардировке чистой поверхности металлов одно- и многозарядными ионами. Предложена простая феноменологическая модель образования атомных ионов, учитывающая релаксацию возбуждения электронной подсистемы в объеме линейного каскада столкновений. В рамках такой модели получены аналитические формулы для расчета вероятности ионизации атомов, распыленных при бомбардировке поверхности металлов одно- и многозарядными ионами. Расчеты по этим формулам показали, что взаимодействие МЗИ с металлом приводит не только к формированию линейного каскада столкновений и локального возбуждения электронной подсистемы в объеме каскада за счет атом-электронных столкновений (как в случае взаимодействия с металлом однозарядных ионов), но и к дополнительному возбуждению электронной подсистемы за счет более эффективных электрон-электронных столкновений. Показано также, что приложение положительного потенциала к поверхности металла приводит к появлению последовательности запаздывающих импульсов возбуждения. Совокупность этих процессов инициирует существенное увеличение вероятности ионизации распыленных атомов за счет более эффективного возбуждения электронов и увеличения времени релаксации этого возбуждения. Полученные результаты имеют практическую значимость, поскольку указывают пути повышения чувствительности и информативности различных модификаций метода масс- спектрометрии вторичных ионов и, возможно, других аналитических методов, использующих эмиссию возбужденных атомов и отрицательных ионов.

\section{Конфликт интересов}

Авторы заявляют, что у них нет конфликта интересов.

\section{Список литературы}

[1] Sputtering by Particle Bombardment, Issue 1, ed. by R. Behrisch (Springer-Verlag, Berlin, 1981)

[2] P. Sigmund. Phys. Rev., 184, 383 (1969). DOI: $10.1103 /$ PhysRev.184.383

[3] S.F. Belykh, B. Habets, U.Kh. Rasulev, A.V. Samartsev, L.V. Stroev, I.V. Veryovkin. Nucl. Instrum. Met. Phys. Res. B, 164-165, 809 (2000) DOI: $10.1016 / \mathrm{S} 0168-583 \mathrm{X}(99) 01079-4$

[4] Sputtering by Particle Bombardment: Experiments and Computer Calculations from Threshold to MeV Energies, ed. by R. Behrisch, W. Eckstein (Springer, Berlin, 2007)

[5] R.G. Wilson, F.A. Stevie, C.W. Magee. Secondary Ion MassSpectrometry: a Practical Handbook for Profiling and Bulk Impurity Analysis. (Wiley, NY., 1989)

[6] Cluster Secondary Ion Mass Spectrometry: Principles and Applications, ed. by C.M. Mahoney (Wiley \& Sons, Hoboken, 2013)

[7] M. Herder, P. Ernst, L. Skopinski, B. Weidtmann, M. Schleberger, A. Wucher. J. Vac. Sci. Technol. B, 38, 044003 (2020). DOI: 10.1116/6.0000171

[8] L. Skopinski, P. Ernst, M. Herder, R. Kozubek, L. Madauß, S. Sleziona, A. Maas, N. Königstein, H. Lebius, A. Wucher, M. Schleberger. Rev. Sci. Instrum., 92, 023909 (2021). DOI: $10.1063 / 5.0025812$ 
[9] N. Nishida, M. Sakurai, D. Kato, H. Sakaue. J. Vac. Sci. Technol. B, 38, 044006 (2020). DOI: 10.1116/6.0000042

[10] A Simple Sputter Yield Calculator. https://www.iap.tuwien.ac.at/www/surface/sputteryield

[11] S.F. Belykh, V.V. Palitsin, A. Adriaens, F. Adams. Phys. Rev. B, 66, 195309 (2002). DOI: 10.1103/PhysRevB.66.195309

[12] M.L. Yu. Charged and Excited States of Sputtered Atoms, in Sputtering by Particle Bombardment III, Eds. R. Behrisch, K. Wittmaack (Berlin, Springer, 1991), p. 91. DOI: $10.1007 / 3-540-53428-8$

[13] H.P. Winter, H. Eder, F. Aumayr, Int. J. Mass Spectrom., 192, 407 (1999). DOI: 10.1016/S1387-3806(99)00074-3

[14] A. Wucher, Z. Sroubek. Phys. Rev. B, 55, 780 (1997). DOI: $10.1103 /$ PhysRevB.55.780

[15] C.K. Sun, F. Vallee, L.N. Acioli, E.P. Ippen, J.G. Fujimoto. Phys. Rev. B, 50, 1537 (1994). DOI: 10.1103/PhysRevB.50.15337

[16] V.P. Zhukov, F. Aryasetiowan, E.V. Chulkov, I.G. Gurtubay, P.M. Echenique. Phys. Rev. B, 64, 195122 (2001). DOI: 10.1103/PhysRevB.64.195122

[17] A.V. Lugovskoy, I. Bray. Phys. Rev. B, 60, 3279 (1999). DOI: 10.1103/PhysRevB.60.3279

[18] С.Ф. Белых, А.Б. Толстогузов, А.А. Лозован, М.Е. Алешин, И.А. Елантьев. ЖЭТФ, 145, 643 (2014). [S.F. Belykh, A.B. Tolstoguzov, A.A. Lozovan, M.E. Aleshin, A. Elantev. J. Experiment. Theoretic. Phys., 118, 560 (2014). DOI: $10.1134 / \mathrm{S} 1063776114030029]$

[19] С.Ф. Белых, А.Б. Толстогузов, А.А. Лозован, М.Е. Алешин, И.А. Елантьев. Поверхность. Рентгеновские, синхротронные и нейтронные исследования, 2, 69 (2015). [S.F. Belykh, A.B. Tolstogouzov, A.A. Lozovan, M.E. Aleshin, I.A. Elantyev. J. Surf. Investigation: X-ray, Synchrotron and Neutron Techniques, 9(1), 172 (2015). DOI: $10.1134 / \mathrm{S} 102745101406024 \mathrm{X}]$

[20] P. Sigmund, C. Claussen. J. Appl. Phys., 990, 52 (1981). DOI: $10.1063 / 1.328790$

[21] С.Ф. Белых, А.Б. Толстогузов, А.А. Лозован. Письма в ЖЭТФ, 101, 712 (2015). [S.F. Belykh, A.B. Tolstoguzov, A.A. Lozovan. JETP Lett., 101, 638 (2015). DOI: 10.1134/S0021364015090064]

[22] Э. Ферми. Молекуль и кристалль (ИЛ, М., 1947)

[23] Z. Sroubek. Phys. Rev. B, 25, 6046 (1982). DOI: $10.1103 /$ PhysRevB.25.6046

[24] Z. Sroubek. Nucl. Instr. Meth., 194, 533 (1982). DOI: $10.1016 / 0029-554 X(82) 90577-8$

[25] E.G. Overbosch, B. Rasser, A.D. Tenner. J. Los. Surf. Sci., 92, 310 (1980). DOI: 10.1016/0039-6028(80)90259-9

[26] G. Betz, W. Husinsky. Nucl. Instrum. Methods Phys. Res. B, 102, 281 (1995). DOI: 10.1016/0168-583X(95)80155-F

[27] A. Arnau, F. Aumayr, P.M. Echenique, M. Grether, W. Heiland, J. Limburg, R. Morgenstern, P. Roncin, S. Schippers, R. Schuch, N. Stolterfoht, P. Varga, T. Zouros, H.P. Winter. Surf. Sci. Rep., 27, 113 (1997). DOI: 10.1016/S0167-5729(97)00002-2

[28] F. Aumayr, H.P. Winter. Phil. Trans. Soc. Lond. A, 362, 77 (2004). DOI: 10.1098/rsta.2003.1300

[29] С.Ф. Белых, А.Д. Беккерман, Д.А. Богуславский, А.Б. Толстогузов. ЖТФ, 91 (1), 120 (2021). [S.F. Belykh, A.D. Bekkerman, D.A. Boguslavskii, A.B. Tolstoguzov. Tech. Phys., 66 (1), 113 (2021).

DOI: $10.1134 / \mathrm{S} 1063784221010023]$ 